\title{
Convergence Performance of SINR and SIR Utilities for a Distributed Resource Allocation GAME
}

\author{
S.S. Prasad ${ }^{1}$, Rithika Baruah ${ }^{2}$ and Siddharth. $A^{3}$ \\ Department of Electronics \& Communication Engineering, National Institute of \\ Technology, Jamshedpur, India \\ ${ }^{1}$ ssprasadeieee.org \\ ${ }^{2}$ rithika_baruah@ieee.org \\ ${ }^{3}$ sidaudhi@gmail. com
}

\begin{abstract}
In ad-hoc networks, the nodes are primarily decentralized in nature. The absence of a central controller because of the decentralization characteristic of these impromptu networks results in autonomous operations for each of the individual users. In such networks, the decisions taken by the users are based on perceived measures or statistics which are collected dynamically. Decision taken by one node affects and influences the other nodes. The scenario of such a network can be optimized using the principles of game theory. The final model of the scenario can be formulated as a non-cooperative game. Algorithms based on this model are devised for ad hoc networks. Applications of such algorithms include pricing schemes for distributed beamforming, bandwidth allocation as well as power control games. Asynchronous Distributed Pricing is a virtual pricing scheme for transmitting power optimization in ad hoc networks. In this algorithm, the difference between the value of a given performance metric and the price paid to attain it determines a user's payoff and the network objective is to maximize the sum of all users' payoff. However, the determination of the utility function essentially determines the global convergence of the sum of all users' payoff. Supermodularity conditions are fundamental constraints for global convergence of utilities in a pricing game which have been previously defined. This paper obtains suitable utility functions satisfying the aforementioned constraints. This paper also demonstrates that performance characteristics of different utilities vary considerably under similar network parameters. This paper also assesses the optimality of utility functions under Signal-to-Interference-plus-Noise ratio as well as Signal-to-Interference ratio based calculations and also explores the difference in performance characteristics obtained by the addition of a significant value of noise variance in the Asynchronous Distributed Pricing algorithm.
\end{abstract}

\section{KEYWORDS}

Game Theory, Asynchronous Distributed Pricing, Distributed Resource Allocation, Supermodularity

\section{INTRODUCTION}

In the emerging ad-hoc technology, a central controller is often absent and operation is thus based mostly on the cooperation between the nodes, which being devoid of a fixed infrastructure, have limitations mostly on their battery power which must be judiciously consumed for transfer of data packets. Resource allocation is hence, being addressed in a distributed framework with power allocation being the most important component of it. Mostly, ad hoc networks find applications in emergency operations and military environment. Figure 1 shows an ad-hoc network and compares it with a traditional cellular network. 
. Figure 1. i) Ad-hoc Network, ii) Traditional Cellular Network

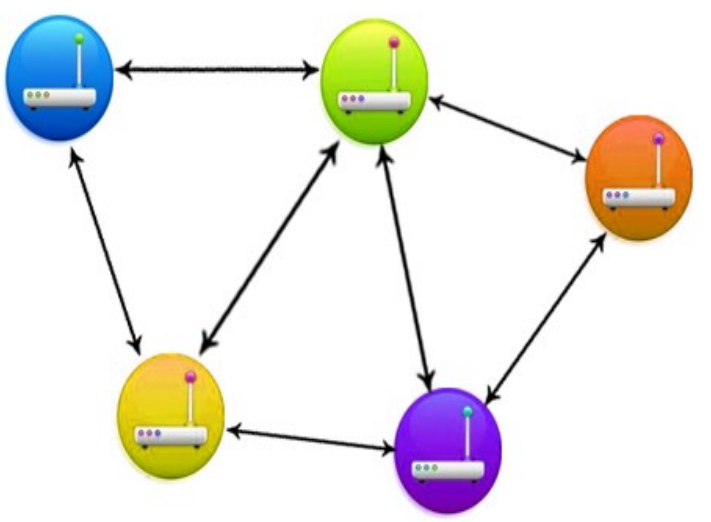

(i)

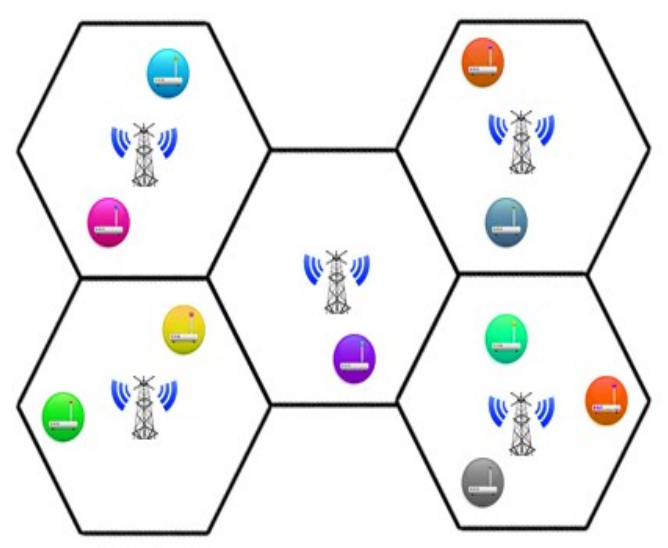

(ii)

Mitigating interference is particularly important in wireless ad hoc networks because of the absence of a central controller or resource manager. Coordination amongst the nodes in such a network is not always necessarily cooperative. A challenge is then to provide a means for coordination to allocate available resources, defined in frequency, space, and time, at each transmitter to avoid interference wherever possible.

Asynchronous Distributed Pricing (ADP) scheme addresses this problem by designing autonomous allocation methods in each transmitter, based on the exchange of virtual interference prices. User demands for service and associated priorities depend on a Quality of Service (QoS) metric. It is known as the utility of the network. The network objective is then the sum utility over all users. This objective is flexible enough to accommodate a wide range of performance metrics through an appropriate assignment of utility functions.

This game theoretical approach to Quality-of-Service (QoS) based distributed resource allocation acts as a preferable alternative to the centralized scheme owing to its advantages of reduced overhead and information exchange. The fundamental objective of this research was to compare the convergence performance of a distributed algorithm, Asynchronous Distributed Pricing (ADP), for various utility functions and Quality-of-Service (QoS) metrics. It was found that the choice of QoS metric determines the different types of performance curves. This study's current model implementing ADP has derived that the choice of QoS metric determines the sum utility analytics.

\section{Related Work}

Game theory is, primarily, a study of mathematical models in order to optimize conflicts and cooperation in an interactive decision making situation. Algorithms for finding solutions of complex interactions among intelligent rational decision makers as defined in [1] consist of various levels of analytical paraphernalia. Rationality, as defined in terms of the players in the game or users/nodes in a wireless network demands a strict adherence to a strategy based on perceived or measured results. Game theory models individual, independent decision makers whose actions potentially affect all other decisions. Hence, the performance of ad hoc networks, in which each node can be treated as a rational, independent, selfish player, can be easily analyzed using game theory. Game theoretic analysis of ad hoc networks has been widely applied and used for power control and waveform adaptation in the physical layer, medium access control as well as routing in the network layer besides others [2, 3]. The pricing 
algorithms have been used for resource allocation games ranging from bandwidth allocation, distributed beamforming, interference pricing algorithms to power control games using virtual currency in [4-7]. Other applications include flow and congestion control and resource sharing in peer-to-peer networks [21 - 22]. In this paper we deal with the problem of network entities chosen for a power control game aiming at efficient resource allocation in ad hoc networks.

The three basic elements of a game set are: rational decision makers, actions and utilities. Rational decision makers are the players in the game who are expected to make decisions based on the rules set by the algorithm in the game in a way so as to gain maximum utility in the game. The alternative courses available to each of the decision makers in order to gain specific utilities accordingly are the actions. The action set chosen determines the final game outcome. In order to optimize the game outcomes, a programmer of a wireless network can design the rules of the algorithm in such a way so as to ensure that the players choose the action sets deriving the maximum utilities. In a typical game, the mathematical entities known as utilities represent desirable outcomes of the game. Higher utilities ensure more desirable outcomes. Games in a wireless network are designed around factors such as a player preferring lower bit error rates, lower transmitting powers and higher signal to noise ratios. In real networks, these goals mostly remain in conflict. Hence, the most intriguing facets of game theory applications are the preference relationships.

When the wireless spectrum is shared by two or more than two users, interference management using efficient resource allocation becomes one of the most important issues as illustrated in [8]. Centralized power allocation, for instance, cellular downlink, is an approach which creates a lot of overhead in the network. However, in ad hoc and mesh networks, distributed approaches are preferred which allocate power using limited information exchange. One particular subtype of game theory is the non-cooperative game theory. In this model the users are assumed to interact using a predefined mechanism while acquiring individual, distinct and well defined interests. Such users formulate strategies in order to maximize their own utilities without being concerned about the overall stability or global utility of the whole system or in this case, the wireless ad hoc network. In such a scenario, the algorithm as the hardwired programming level is designed so as to form a game based on rules which ensure fair play, i.e., maximum global utility without individual incentives. This game theoretical model basically consists of fields in applied mathematics such as normal and extensive form games, incomplete information, Nash equilibrium, and forward and backward induction. Hence, non-cooperative game theory can be used to solve this physical layer issue of adjusting transmitting power levels in order to adjust interference in the network keeping it below a threshold level beyond which interference becomes a significant problem $[9,10]$.

Within the game theoretic framework, the entities in the wireless network are as follows: players of the game are the nodes, i.e., transmitter-receiver pairs; utility function for the game is the performance metric or quality-of-service (QoS) metric of the users and strategy of the game is the algorithm or approach adopted for the network. The strategy in a power game for wireless networks is devised in such a way that the sum utility for all the players of the game is maximized within the least number of iterations. This strategy can either be centralized or distributed. Our paper considers efficient resource allocation in ad hoc networks and hence, we deal with distributed algorithms. Shi et al. in [11] have shown that Asynchronous Distributed Pricing (ADP) converges within the least possible number of iterations as compared to previous distributed algorithms.

However, one important entity in the ADP is the choice of utility function because it determines the convergence of the algorithm. At Nash equilibrium of a game, both the power and price player chose not to deviate. The convergence of ADP algorithm, thus, can be ascertained by showing that the best response updates of the game converge. Nash equilibrium may or may not 
International Journal of Wireless \& Mobile Networks (IJWMN) Vol. 3, No. 3, June 2011

exist in all arbitrary games and even if it does exist, best response updates need not converge to it. However, for the class of supermodular games defined and explained in [12], best response updates converge even when the algorithm for power or price update is arbitrarily asynchronous. ADP has been proved to contain a global optimum for supermodular games in [13].

Schmidt et. Al in [12] have proved that the coefficient of relative risk aversion decides the convexity of a utility function and hence, its choice to be used in the algorithm. However, this paper shows that the noise parameter when incorporated significantly changes the performance characteristics for various utilities lying within stated constraints of coefficient of relative risk aversion. In this paper, we deal with the rate of convergence of the algorithm for a significant noise variance and compare the characteristics for Signal-to-Interference-Ratio (SIR) and Signal-to-Interference-plus-Noise-Ratio (SINR) based calculations.

In the following sections, the problem of the distributed wireless resource allocation game is formulated as a non-cooperative game, the mathematical model for the problem within an assumed system framework is defined and the final results of the research are presented and explained.

\section{Problem Formulation}

Asynchronous Distributed Pricing is a distributed algorithm and hence, each node acts autonomously and each node updates its utility asynchronously according to the network statistics. The network objective in this problem is to find a global optimum solution for the maximum sum utility over all users. This objective accommodates a wide range of QoS metrics. This can be done by assigning the utility functions accordingly.

Let us first consider the characteristics of this problem which makes it apposite to be used as a non-cooperative game theoretical problem.

1. Each user makes its own decision autonomously based on 'selfish' utilities.

2. The users' utilities, i.e., SINR and SIR, are functions of their own payoffs.

3. Payoffs of the players are functions of their own transmit levels which are controlled according to the algorithm used.

4. Increase in the transmitting power level of a node/user decreases the SINR/SIR of other nodes/players, thus creating conflict of interests in the game.

5. Nodes prefer lower transmitting power levels for a fixed SINR/SIR and higher SINR/SIR for a fixed transmitting power level.

The primary convergence rule for such game models depends upon the existence of Nash equilibrium. Basically, Nash equilibrium is defined as a joint strategy in a game where no player can increase its own utility by deviating unilaterally. Mathematically,

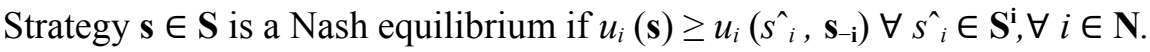


International Journal of Wireless \& Mobile Networks (IJWMN) Vol. 3, No. 3, June 2011

In networks offering different levels of QoS, network performance will be directly influenced by the users' decisions about the level of service to request. Network profitability or some social welfare goal such as the sum of users' payoffs can be maximized by designing virtual pricing algorithms. Virtual price is the cost paid by the users in order to increase their own utilities while degrading the utilities of other users. Each user's QoS choice may, thus, be influenced not only by the pricing policy but also by other users' behavior. The problem can naturally be treated under as a game defined by the operating point predicted by its Nash equilibrium.

Pricing can be treated as a game between a network service provider and a finite set of nodes. Different users have different QoS requirements. Each user must choose its own strategy and level of QoS. For example, price or cost can be dependent on a number of factors such as battery power, transmission power and throughput, specific to a problem [21]. In priority-based networks, strategy may be the priority levels a user requests for her traffic. Let us take the example of networks supporting delay; it ensures a strategy of minimum bandwidth. The tradeoff is that the higher the level of QoS requested, the higher the price to be paid by the user. The network service provider drafts the Nash equilibrium by setting the rules of the game and designing its pricing structure.

A user's payoff is the difference between how much a user values a given QoS and how much is the price paid for it. Rationally, optimum strategy of each user is derived by the maximization of this payoff, given all other users' service choices remain. Every receiver declares its own interference price in the network which indicates the marginal decrease in utility due to a marginal increase in interference associated with a particular Degree of Freedom (DoF). A transmitter selects power according to a best response, which maximizes its utility minus the cost of interference incurred. Users iterate between price and power updates until the algorithm has converged. Convergence signifies that when the best response using the payoff function is obtained, the transmitted powers and interference prices do not change in subsequent iterations. Moreover, ADP algorithm's superiority lies in its advantage of fast convergence. While gradient based algorithms may take around 80 iterations to converge, ADP takes only 2-4 as proved in $[14,15]$.

In [16], it has been stated that SIR and SINR balancing is fundamental for characterization of QoS feasible region in wireless network problems. Although convergence analysis in previous papers [14-16] has revolved around supermodular games only, we have observed that the utilities lying within the constraint defining supermodular games also vary in their performance characteristics. The amount of concavity of a function determines its usability in the ADP algorithm but an important question to explore is the variation of the convergence parameters with varying utility functions as well as SIR and SINR based calculations.

In the next section, the system framework in which the ad hoc network is simulated is discussed and illustrated along with a detailed mathematical model of the algorithm.

\section{System Framework and Mathematical Perspective}

We consider the following assumptions for the problem. Each "user" in the network is a single transmitter/receiver pair. Each transmitter uses the same bandwidth. Each receiver is interested in the signal from its associated transmitter only. The disturbance to the message signals comes from all other transmitters which constitute the interference. In addition to interference, all the receivers experience equal amount of background noise. The conditions of the wireless channel are reflected in the channel gains between each transmitter and each receiver. 
We have considered a system of 3 transmitter-receiver pairs with channel matrices $h_{i j}$ where $i$ is the receiver and $\mathrm{j}$ is the transmitter. The system model is shown in Fig 2.

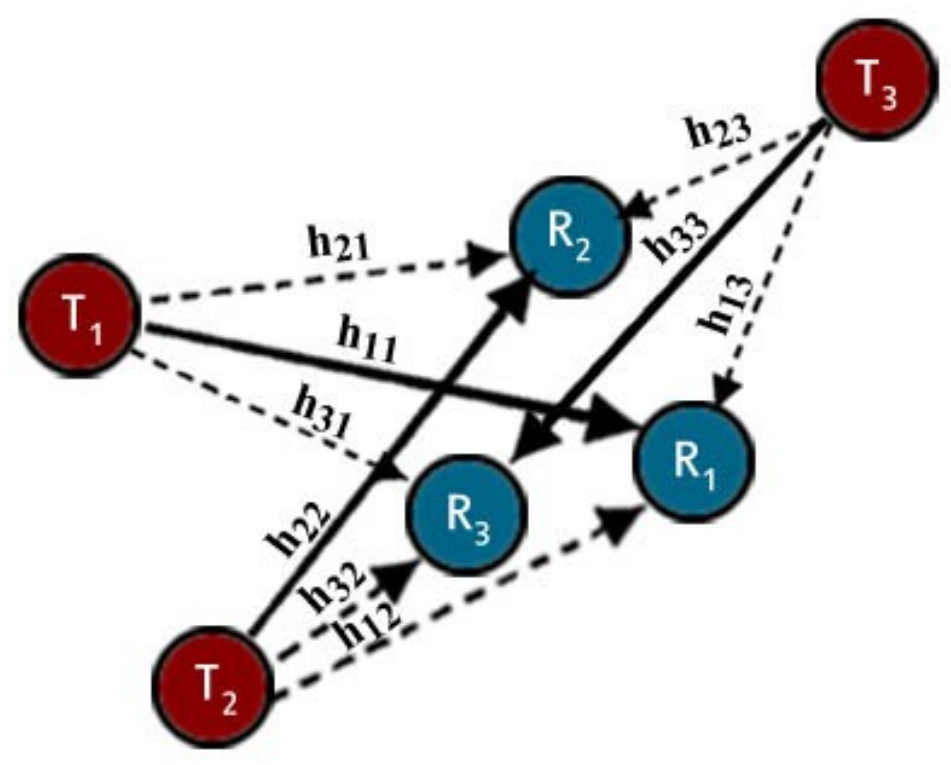

. Figure 2. System framework

We assume perfect channel estimation for all the nodes in our model. The channel is modeled as a lossy channel for SINR calculations and SIR calculations. Each user needs to know adjacent channel gains and interference prices only. This decreases the overall information exchange overhead. User's QoS are preferences given by a utility function $u_{i}\left(R_{i}(P)\right)$ where $u_{i}(\cdot)$ is increasing, twice differentiable and sufficiently concave function of $\mathrm{R}_{\mathrm{i}}$. Sufficient concavity is defined in [14] which is modeled in supermodular games relies on the constraint that the utility must neither be non-concave nor too concave.

Common QoS metrics used to perceive a user's performance in a network are the received SIR and SINR. ADP algorithm utilizes a virtual currency scheme to localize the optimization problem faced in maximizing the sum utility of the network, by allowing the nodes to autonomously solve the power optimization problem constrained by the strategy of the game and maximum of the principal entity which is the transmitting power in a power game.

The resulting payoff function for power optimization suggested by the algorithm is,

$$
\Pi\left(P_{i}, \pi_{j}\right)=u_{i}-\sum_{j \neq i} \pi_{j} h_{j i}^{2} P_{i}
$$

where $\prod$ is the net payoff, $u_{i}$ is the utility function, $\prod_{i}$ is the price announced by the $j^{\text {th }}$ receiver, $h_{j i}$ is the cross channel gain between the $i^{t h}$ transmitter and the $j^{t h}$ receiver and $P_{i}$ is the power transmitted by the $i^{\text {th }}$ transmitter.

The interference price is a virtual quantity which is the marginal cost of a user's own utility per unit interference as is given by: 


$$
\pi_{i}=-\frac{d}{d I_{i}}\left(u_{i}\right)
$$

The choice of utility function is essential for the convergence of the algorithm. In [17], J. Yuan and $\mathrm{W}$. Yu have shown that the ADP power game becomes supermodular if the coefficient of relative risk aversion factor lies between 1 and 2, resulting in a global optimum solution for the sum utility.

We chose two main utility functions, which satisfy the necessary condition of supermodularity, $\log (\mathrm{x})$ with $\mathrm{CR}_{\mathrm{k}}(\mathrm{x})=1$ and $-1 / \mathrm{x}$ with $\mathrm{CR}_{\mathrm{k}}(\mathrm{x})=2$ and compared their performance with that of the rate utility $\log (1+\mathrm{x})$ for which $\mathrm{CR}_{\mathrm{k}}(\mathrm{x})<1$. However, in [18] it has been shown that rate utilities also converge subject to a few constraints on the ADP algorithm. We consider a diagonally dominant channel and the convergence thus obtained is a local optimum which may be multiple depending upon the test cases considered. Also, we compared the results of the utilities for both SIR and SINR service metrics.

\subsection{Case $I: u(x)=\log (x)$}

As the optimization problem is solved locally, for individual nodes, the variable parameter is $P_{i}$

$$
\prod\left(P_{i}, \pi_{j}\right)=\log \left(a_{i} P_{i}\right)-\sum_{j \neq i} \pi_{j} h_{j i}^{2} P_{i}
$$

$$
\begin{gathered}
\qquad\left(P_{i}, \pi_{j}\right)=\log \left(a_{i} P_{i}\right)-b_{i} P_{i} \\
\text { where } a_{i}=\frac{h i i^{2}}{\sum_{j \neq i} h i j^{2} P_{j}+\sigma^{2}} \text { and } b_{i}=\sum_{j \neq i} \pi_{j} h_{j i}^{2}
\end{gathered}
$$

Maximizing (3), the condition for power update in ADP algorithm is,

$$
\boldsymbol{P}_{i}=\frac{1}{b_{\mathrm{i}}}
$$

It is notable that the interference price broadcasted by the user is equal to the interference power seen by it. Price of SINR based utility functions is calculated as the inverse of the sum of interference power and the noise variance at the receiver $\left(\sigma^{2}\right)$.

$\boldsymbol{\pi}_{i}= \begin{cases}\frac{1}{I_{1}} & \text { for SIR based } \\ \frac{1}{I_{1}+\sigma^{2}} & \text { for SINR based }\end{cases}$

\subsection{Case II: $u(x)=-1 / x$}

Re-writing (1) in terms of the local variable $P_{i}$ 
International Journal of Wireless \& Mobile Networks (IJWMN) Vol. 3, No. 3, June 2011

$$
\begin{gathered}
\Pi\left(P_{i}, \pi_{j}\right)=-\frac{1}{a_{i} P_{i}}-b_{i} P_{i} \\
\text { where } a_{i}=\frac{h i i^{2}}{\sum_{j \neq i} h i j^{2} P_{j}+\sigma^{2}} \text { and } b_{i}=\sum_{j \neq i} \pi_{j} h_{j i}^{2}
\end{gathered}
$$

Maximizing (4),

$$
=>P_{i}=\frac{1}{\sqrt{a_{i} b_{i}}}
$$

The parameter $a_{i}$ incorporates the noise variance factor in case of SINR based utility function. The interference price of the user is found to be independent of its interference power and noise, and is given by:

$$
\pi_{i}=\frac{1}{h_{i i}^{2} P_{i}}
$$

When choosing SIR as a service metric for measuring the performance of a system and for power optimization, it is to be noted that the solution arising from the algorithm may not be universal. The resulting SIR, being a ratio of powers which are optimized by the algorithm, may not be unique. In a three user system, if the solution of the SIR based algorithm gives rise to three powers $\mathrm{P}_{1}, \mathrm{P}_{2}$ and $\mathrm{P}_{3}$, then the set of powers $k \mathrm{P}_{1}, k \mathrm{P}_{2}$ and $k \mathrm{P}_{3}$, where $k$ is a constant, also give the same SIR. Therefore, the converged value of transmission powers obtained through one utility function may differ from that obtained from another, depending on the initial state of the algorithm.

In case of SINR based utility functions, the solution obtained through the algorithm is unique, owing to the noise variance factor in the denominator of the service metric. The existence of the noise variance parameter increases the number of iterations required for convergence. Comparing the sum utilities after convergence of SIR based and SINR based algorithms, it is evident that SINR based utility functions give a better performance than their SIR based counterparts, due to the incorporation of the noise variance parameter, though consuming larger time.

In this paper, we have already mentioned that the utilities of the final game change considerably when the payoff metric is changed. The proof of the research has been proved mathematically in the previous section and the final section discusses the results of the simulations performed in order to establish our mathematical derivations and verifications. The next section elaborates the test cases which were developed to perform the necessary simulations.

\section{Test Cases}

To evaluate the performance of SIR based and SINR based utility functions in ADP algorithm, we chose the following test cases:

Table 1. Test Cases for Simulation.

\begin{tabular}{|l|l|l|}
\hline Case & QoS Metric & Utility function \\
\hline 1 & Signal-to-Interference Ratio (SIR) & $\mathrm{u}(\mathrm{x})=\log (\mathrm{x})$ \\
\cline { 3 - 3 } & & $\mathrm{u}(\mathrm{x})=-1 / \mathrm{x}$ \\
\hline 2 & $\begin{array}{l}\text { Signal-to-Interference-plus-Noise- } \\
\text { Ratio(SINR) }\end{array}$ & $\mathrm{u}(\mathrm{x})=\log (\mathrm{x})$ \\
\cline { 3 - 3 } & $\mathrm{u}(\mathrm{x})=-1 / \mathrm{x}$ \\
\hline
\end{tabular}


In order to compare the performance of the two cases, we assumed that the algorithm was initiated by the same set of arbitrary powers, prices and channel gains.

Simulation results have been recorded and interpreted in the next section which is followed by the conclusions and future scope of this paper.

\section{Observations and Results}

The simulation of the ADP algorithm for the test cases mention in the previous section found that the algorithm converged for all the test cases. The negation of noise variance parameter leads to premature convergence of the algorithm thereby reducing the sum utility of the system. At the same time it is also observable from Fig. 2 that the SIR based $-1 / x$ utility function provided a much better compared to that of SIR based $\log (\mathrm{x})$, which was very close to the solution attained through the rate utility function.

When choosing SIR as a service metric for measuring the performance of a system and for power optimization, the solution arising from the algorithm may not be universal. The resulting SIR, being a ratio of powers which are optimized by the algorithm, may not be unique. In a three user system, if the solution of the SIR based algorithm gives rise to three powers $\mathrm{P}_{1}, \mathrm{P}_{2}$ and $\mathrm{P}_{3}$, then the set of powers $k \mathrm{P}_{1}, k \mathrm{P}_{2}$ and $k \mathrm{P}_{3}$, where $k$ is a constant, also give the same SIR. Therefore, the converged value of transmission powers obtained through one utility function may differ from that obtained from another, depending on the initial state of the algorithm.

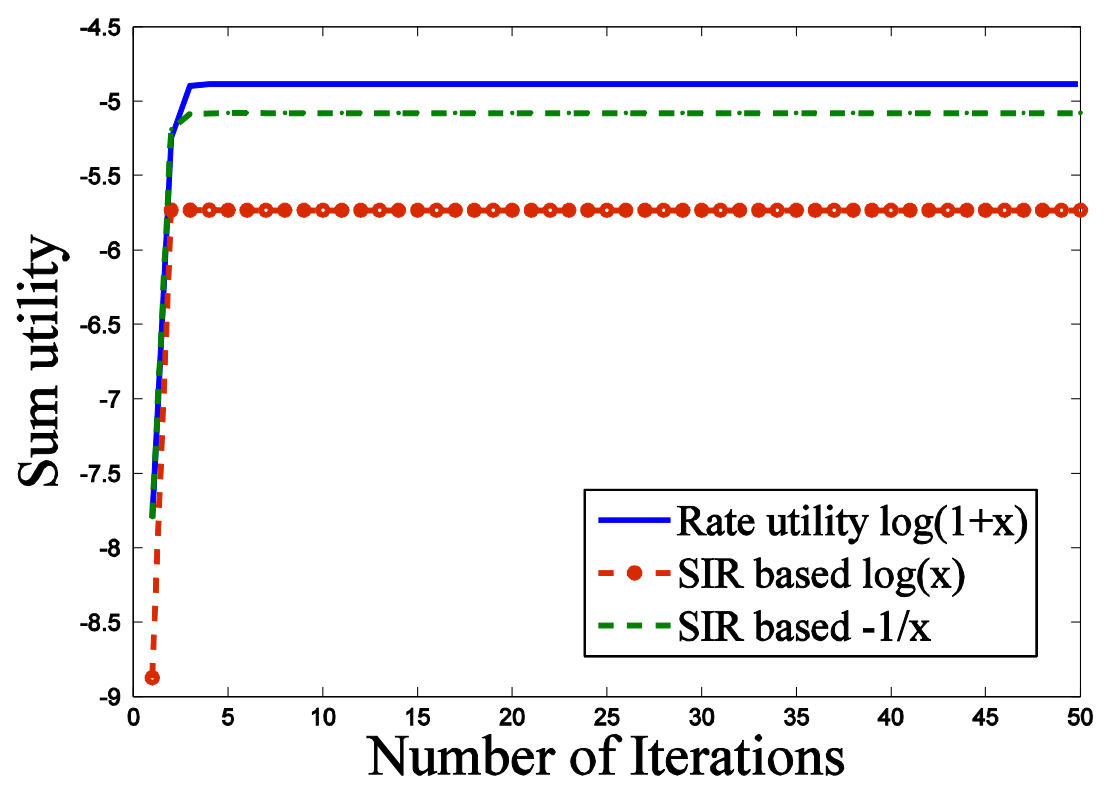

. Figure 2. Comparison of rate utility, SIR based $\log (\mathrm{x})$ and-1/x utility function

The inclusion of noise variance parameter by SINR based utility functions allowed the ADP algorithm to converge to a relatively higher sum utilities as compared to both the rate utility function $\log (1+$ SIR $)$ and SIR based utilities. It is evident from Fig. 3 that the solution of the power game using the two chosen utility functions $\log (\mathrm{x})$ and $-1 / \mathrm{x}$, exhibiting supermodularity, converged to a globally optimum solution. 


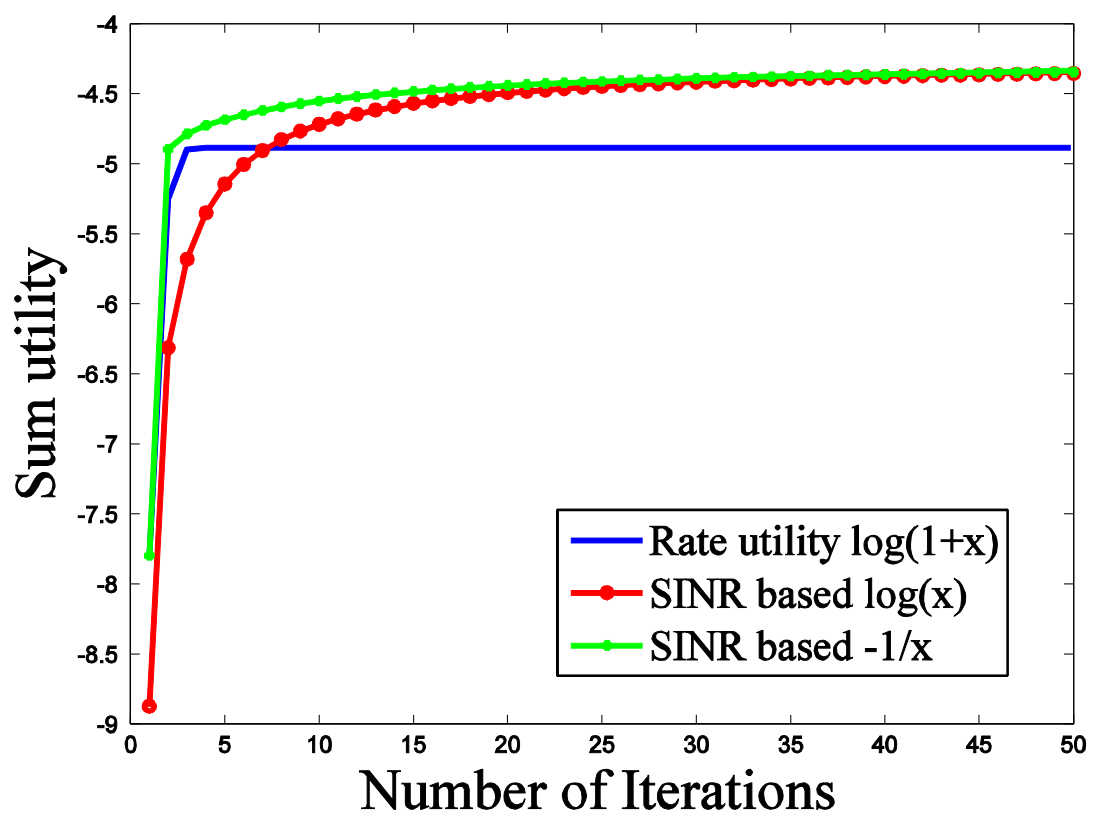

. Figure 3. Comparison of rate utility, SINR based $\log (\mathrm{x})$ and-1/x utility function

This globally optimum solution is better than that obtained through the rate utility function $\log (1+$ SINR $)$. It can also be observed that the $-1 / x$ sum utility curve attains values closer to the optimal solution when compared with $\log (\mathrm{x})$ sum utility. In case of SINR based utility functions, the solution obtained through the algorithm is unique, owing to the noise variance factor in the denominator of the service metric. The existence of the noise variance parameter increases the number of iterations required for convergence.

Figures 2 and 3 provide a comparative study of SINR and SIR based $-1 / \mathrm{x}$ and $\log (\mathrm{x})$ utility functions respectively. $-1 / \mathrm{x}$ utility function provides a much better convergence when compared with $\log (\mathrm{x})$ utility function. The implementation of $\log (\mathrm{x})$ utility function in ADP algorithm is a popular technique for obtaining a sufficiently concave function of sum of utilities [19], owing to its close association with the rate-utility function corresponding to the Shannon capacity of the channel. However, we observe that the $-1 / \mathrm{x}$ utility function gives a better sum utility than $\log (\mathrm{x})$ in SIR based calculations although it converges to a value similar to $\log (\mathrm{x})$ in the SINR based calculations. In the comparison between the sum utility curves of ADP algorithm using SINR based and SIR based utility functions, it is notable that SINR based utility functions offer better performance. But comparing individual utility functions with SIR and SINR arguments, the ADP algorithm using SINR based utility functions require larger number of iterations for convergence; whereas ADP algorithm using SIR based utility functions, converge faster.

\section{Conclusions}

QoS based distributed resource allocation is preferred over to the centralized scheme for ad hoc networks. Advantages of distributed algorithms are their abilities to exist and operate without the requirement of a central controller along with the significantly reduced overhead and information exchange. Using the Asynchronous Distributed Pricing (ADP) algorithm, this paper and found that the choice of QoS metrics and the utility functions drives the performance characteristics of the convergence curves. Moreover, our model implementing ADP derives that using SINR based utility functions provides a much better sum utility when compared to SIR 
International Journal of Wireless \& Mobile Networks (IJWMN) Vol. 3, No. 3, June 2011

based utility functions in lossy channels. Comparing utility functions $\log (\mathrm{x})$ and $-1 / \mathrm{x}$, this paper finds that $-1 / \mathrm{x}$ provides a much higher sum-utility and convergence. The advantage of SIR based utility functions lies in its faster convergence although to a relatively poorer solution with respect to SINR based utility functions. SINR, when used as a metric in the rate utility function of $\log (1+\mathrm{SINR})$ for the ADP algorithm, is observed to converge to a locally optimal solution, due to the non-uniqueness of the set of powers obtained in the solution. This result is consistent with the previous papers which base this observation on the coefficient of relative risk aversion. Hence, this paper finds that while SIR leads to quicker convergence, the inclusion of the noise variance parameter in the SINR, allows the ADP algorithm to converge to a higher utility globally optimum solution. Additionally, $-1 / \mathrm{x}$ utility function results in higher sum utilities and therefore, provides better convergence performance as compared to $\log (\mathrm{x})$ utility or rate utility $\log (1+\mathrm{x})$ functions. The future scope of this work lies in interference management in the given channel conditions in order to ensure higher convergence utilities.

\section{REFERENCES}

Roger B. Myerson, "Game Theory: Analysis of Conflict”, Harvard University Press, 1991.

J. Hicks, A. MacKenzie, J. Neel, J. Reed, "A game theory perspective on interference avoidance," in IEEE Global Telecommunications Conference., vol. 1, pp. 257-261, December 2004.

V. Srivastava, J. Neel, A.B. Mackenzie, R. Menon, L.A. Dasilva, J.E. Hicks, J.H. Reed, and R.P. Gilles, "Using game theory to analyze wireless ad hoc networks," in IEEE Communications Surveys \& Tutorials, vol. 7, no. 4, pp. 46-56, 2005.

Y. Qiu and P. Marbach, "Bandwidth allocation in ad hoc networks: A price-based approach," in Proceedings of IEEE INFOCOM, vol. 2, 2003, pp. 797-807.

Y. Xue, B. Li, and K. Nahrstedt, "Price based resource allocation in wireless ad hoc networks," in Proceedings of 11th International Workshop on QoS, 2003.

C. Shj and M.L. Honig, "Local interference pricing for distributed beamforming in MIMO networks," in Proc. Military Communications Conference, 2005, pp. 1-9, Oct 2009.

C. Shi, D. A. Schmidt, R. A. Berry, M. L. Honig, and W. Utschick, "Distributed Interference Pricing for the MIMO Interference Channel," in Proc. IEEE International Conference on Communications, June 2009.

V. V. Veeravalli, "Interference Management in Wireless Networks," in Qualcomm Seminar, San Diego, CA, February 2008.

S. Agarwal, S. Krishnamurthy, R. Katz, and S. Dao, "Distributed power control in ad-hoc wireless networks," in International Symposium Personal, Indoor and Mobile Radio Communications, pp. F-59-F-66, 2001.

J. Huang, R. A. Berry, and M. L. Honig, "Performance of Distributed Utility-Based Power Control for Wireless Ad Hoc Networks," in Proceedings of Military Communications Conference 2005, pp. 2481-2487, Oct 2005.

C. Shi, R.A. Berry, and M.L. Honig, "Distributed interference pricing for OFDM wireless networks with non-separable utilities," in Proceedings of Conference on Information Sciences and Systems 2008, pp. 755-760, March 2008.

D. Schmidt, C. Shi, R.A. Berry, M. Honig, and W. Utschick, "Distributed Resource Allocation Schemes," in IEEE Signal Processing Magazine, vol. 26, no. 5, pp. 53-63, September 2009.

D. M. Topkis, "Supermodularity and Complementarity," Princeton University Press, 1998.

J. Huang, R. A. Berry, and M. L. Honig, "Distributed interference compensation for wireless networks," in IEEE Journal on Selected Areas in Communications, vol. 24, no. 5, pp. 1074-1084, May 2006. 
International Journal of Wireless \& Mobile Networks (IJWMN) Vol. 3, No. 3, June 2011

C. Shj and M.L. Honig, "Monotonic convergence of distributed interference pricing in wireless networks," in IEEE International Symposium on Information Theory 2009, pp. 1619-1623, JuneJuly 2009.

Martin Schubert and Holger Boche, "QoS-Based Resource Allocation and Transceiver Optimization," in Foundations and Trends in Communications and Information Theory 2005, vol. 2, no. 6, p.383529, November 2005.

J. Yuan and W. Yu, "Distributed cross-layer optimization of wireless sensor networks: A game theoretic approach," in Proc. IEEE Conf. Global Communications, San Francisco, CA, Nov. 2006.

H. Boche, S. Naik, and T. Alpcan, "Concave resource allocation problems for interference coupled wireless systems," in IEEE International Conference on Acoustics Speech and Signal Processing 2010, pp. 3002-3005, March 2010.

S. Stanczak, M. Wiczanowski and H. Boche, "Distributed Utility-Based Power Control: Objectives and Algorithms," in IEEE Transactions on Signal Processing, vol. 55, no. 10, pp. 5058-5068, October 2007.

T.V.P. Sundararajan and A.Shanmugam, "Self Avoidance Routing Protocol for Mobile Ad Hoc Network" in International Journal of Wireless and Mobile Networks, vol. 2, no. 2, pp. 80-92, May 2010.

E. Altman, "Flow control using the theory of zero sum Markov games", in IEEE Transactions on Automatic Control, vol. 39, no. 4, pp. 814-818, April 1994

Ao Tang and L.H.Andrew, "Game Theory for Heterogenous Flow Control", in Proc. of 42 ${ }^{\text {nd }}$ Annual Conf. on Information Sciences an Systems, pp. 52-56, March 2008

\section{Authors}

Dr. Shyam Sundar Prasad is an Asst. Professor at National Institute of Technology, Jamshedpur, India. He has 24 papers to his credit published in various International, National Journals and Conference Proceedings of repute among which 7 papers have been published in various IEEE proceedings. He has written 4 articles/chapters for books. He is a Senior Member of IEEE (USA), Life member ISTE, Branch Counsellor for NIT Jamshedpur IEEE Student Branch. Presently he is an FPM Scholar at XLRI, Jamshedpur, India.

Rithika Baruah pursued her B.Tech from the Department of Electronics and Communication Engineering at National Institute of Technology Jamshedpur. She has presented 2 papers in National Conferences and published 1 paper in the Proceedings of an International Conference available in IEEEXplore. She is a member of the IEEE and had previously held the position of the Chairperson of NIT Jamshedpur IEEE Student Branch. Her current research interests in wireless communications include Game Theory, Ad hoc networks and Cognitive Radio.

Siddharth A completed his B.Tech from the Department of Electronics and Communication Engineering at National Institute of Technology Jamshedpur. He has presented a paper at a National Conference for Communication. He is member of the IEEE and a part of the IEEE Student Branch at NIT Jamshedpur. His current research interests include CDMA repeaters and Game Theory for Wireless Resource Allocation.
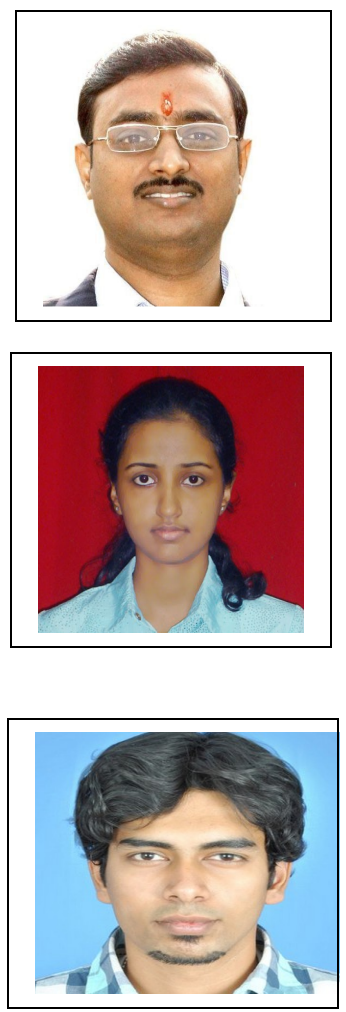